\title{
Preface
}

\section{EXOPLANET Special Issue}

\author{
Nader Haghighipour, Guest Editor September 30, 2014
}

Astrobiology embraces the search for potentially inhabited planets within our Solar System and beyond. To that end, the search for extrasolar planets and exoplanetary science is an integral part of astrobiology.

The past decade has been the era of some of the greatest advances in exoplanetary science. The successful operation of missions such as CoRoT, Spitzer and Kepler space telescopes, combined with the findings by large ground-based surveys, including High Resolution Echelle Spectrometer (HIRES) ${ }^{1}$ and High Accuracy Radial Velocity Planetary Search Project (HARPS) ${ }^{2}$ have provided us with a wealth of confirmed and candidate planetary bodies, each with their unique characteristics. The diversity of these objects in dynamics and physical properties has opened many new chapters in planetary science and has resulted in the development of many new models and theories that have consequently re-shaped our understanding of the formation and evolution of planetary systems in ways unimaginable before. This special edition offers a collection of several papers on this cutting-edge research.

Plans for publishing this special issue were conceived in the early 2014. A number of authors who are well recognized in their respective fields of research were then invited to submit original research articles on a variety of areas in exoplanetary science. This resulted in a collection of 16 papers covering topics from analytical celestial mechanics, to planet formation and migration, data analysis, detection technique, atmospheric and interior properties, future missions and hypotheses papers.

During the preparation of this special issue, I had the pleasure of working with many great individuals without whom this compilation of works would not have materialized. I am grateful to all the authors for accepting my invitation and for their responsiveness during the refereeing and editorial processes. Many thanks also go to all anonymous referees for their outstanding contributions, with each paper having been refereed by an anonymous referee and by myself. I am also deeply grateful to Ms Amanda Johns from the Cambridge University Press for her tremendous help in receiving manuscripts, working with authors during revisions and finalizing papers for publication. This publication would not have been possible without her help. Finally, I would like to thank Dr Rocco Mancinelli, the Chief Editor of the International Journal of Astrobiology for proposing the idea of this special issue and for inviting me to serve as its Guest Editor. 